\title{
Terahertz tunnel ionization of DX-centers in AlGaAs : Te
}

\author{
H. Ketterl ${ }^{\mathrm{a}, *}$, E. Ziemann ${ }^{\mathrm{a}}$, S.D. Ganichev ${ }^{\mathrm{a}, \mathrm{b}}$, I.N. Yassievich ${ }^{\mathrm{b}}$, A. Belyaev, \\ S. Schmult ${ }^{\mathrm{a}}, \mathrm{W}$. Prettl ${ }^{\mathrm{a}}$ \\ anstitut für Exp. und Angew. Physik, Universität Regensburg, 93040 Regensburg, Germany \\ b A.F. Ioffe Physicotechnical Institute, RAS, St. Petersburg, 194021, Russia \\ ${ }^{\mathrm{c}}$ Institute of Semiconductor Physics, NASU, Kiev, 252028, Ukraine
}

\begin{abstract}
Ionization of DX-centers in AlGaAs: Te has been investigated in strong terahertz electric fields of FIR-laser radiation with photon energies much smaller than the impurity binding energy. Detachment of electrons from DX-centers is caused by phonon-assisted tunneling being independent of the field frequency as long as the tunneling time is smaller than the field period. In the opposite case an enhancement of the emission probability with rising frequency has been observed. At very high-field strengths direct tunneling without involving phonons dominates and finally emission rates get frequency independent. (C) 1999 Elsevier Science B.V. All rights reserved.
\end{abstract}

Keywords: DX-center; Persistent photoconductivity; Ionization; Tunneling

\section{Introduction}

The ionization of DX-centers in AlGaAs: Te in a strong terahertz electric field of a powerful far-infrared laser has been investigated. Although the quantum energy of radiation is much smaller than the binding energy of the impurities, a persistent photoconductive signal (PPC) due to the detachment of electrons from the DX-centers has been observed. In a wide range of temperatures, electric field strengths and frequencies, the emission process can be attributed to phonon-assisted tunneling, Refs. [1,2]. In contrast to tunneling ionization of atoms, where only electron tunneling takes place, ionization of impurities in solids is accomplished by two simultaneous tunneling processes, electron tunneling and the redistribution of the vibrational system by defect tunneling. Within a broad range of intensities and wavelengths, the terahertz electric field of the exciting

\footnotetext{
* Corresponding author. Tel.: 0049-0-941-943-3301; fax: 00490-941-943-2177.

E-mail address: hermann.ketterl@physik.uni.regensburg.de (H. Ketterl)
}

radiation acts like a static field. In the case $\omega \tau<1$, where $\omega$ is the radiation frequency and $\tau$ is the tunneling time, electrons tunnel at constant energy through the barrier formed by the impurity binding potential and that of the high-frequency electric field. The ionization probability is independent of frequency and increases with rising field strength $E$ like $\exp \left(E^{2} / E_{\mathrm{c}}^{* 2}\right)$ where $E_{\mathrm{c}}^{*}$ is a characteristic field. At higher frequencies and lower temperatures $\omega \tau>1$ can be achieved, which leads to an increase of the ionization rate by an enhancement of the tunneling probability. In this high-frequency limit electrons can absorb energy from the radiation field during tunneling and thus leave the potential barrier at a higher energy level. By this the effective width of the tunneling barrier is reduced and thus, the tunneling probability enhanced. The ionization probability is characterized by the same field dependence, as in the quasi-static regime $\omega \tau<1$, but substantially increases with increasing frequency. With increasing electric field strength direct carrier tunneling from the bound state into continuum, without participation of phonons, becomes dominant. Here, a less strong dependence of the ionization probability on the electric field strength is observed and the frequency dependence practically vanishes at very high fields. 


\section{Experimental technique}

The experimental investigations presented here have been carried out on DX-centers in $\mathrm{Al}_{0.35} \mathrm{Ga}_{65} \mathrm{As}$ : Te, Refs. $[3,4]$. Samples have been cooled that at thermal equilibrium practically all carriers were bound to deep impurities ( $T=4.2-150 \mathrm{~K})$. Terahertz electric fields have been applied using high-power FIR lasers pulses. The laser was a line-tunable $\mathrm{NH}_{3}$-laser optically pumped by a high-power TEA- $\mathrm{CO}_{2}$-laser, Ref. [5]. Electric field strengths in the semiconductor samples up to about 40 $\mathrm{kV} / \mathrm{cm}\left(\simeq 5 \mathrm{MW} / \mathrm{cm}^{2}\right)$ could be achieved in the frequency range from 5 to $50 \mathrm{THz}$ with $40 \mathrm{~ns}$ laser pulses. The ratio of irradiated conductivity $\sigma_{\mathrm{i}}$ and dark conductivity $\sigma_{\mathrm{d}}$ has been determined from peak values of photoconductive signals using a standard $50 \Omega$ load resistor circuit, Ref. [5].

\section{Results and discussion}

Irradiation of the samples with FIR-radiation leads to positive persistent photoconductivity. Persistent photoconductivity can be attributed to the detachment of electrons from the DX-centers, Refs. [3,6,7], caused by simultaneous electron tunneling in the electric field of the radiation and tunneling redistribution of the defect vibrational system, shown in Fig. 1. In semiclassical approximation the probability of this process may be written as, Ref. [8],

$e(E)=\iint P_{\mathrm{e}} P_{\mathrm{d}} \exp \left(-\mathscr{E} / k_{B} T\right) \mathrm{d} \varepsilon \mathrm{d} \mathscr{E}$,

where $P_{\mathrm{e}}$ and $P_{\mathrm{d}}$ are the electron and the defect tunneling probability, respectively, $T$ is the temperature, $\varepsilon$ the electron energy at tunneling (see inset in Fig. 3) and $\mathscr{E}$ the corresponding defect tunneling energy (Fig. 1). The Boltzmann factor takes into account the thermal excitation of the system in the adiabatic potential $U_{1}$. For the

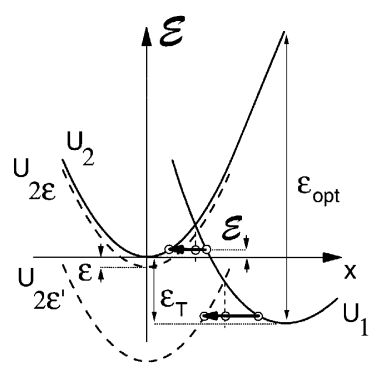

Fig. 1. Adiabatic potential configurations. The energy of vibration and electron is shown as a function of a configuration coordinate $x$. Potentials plotted in broken lines correspond to the electron with negative kinetic energy tunneling in electric fields of two different strengths. electron energy smaller than the defect tunneling energy and the thermal binding energy, $\varepsilon \ll \varepsilon_{\mathrm{T}}$, it has been shown that the dependence of the ionization probability on the alternating electric field strength $E$ is given by, Ref. [8],

$e(E) \propto \exp \left[\frac{E^{2}}{\left(E_{\mathrm{c}}^{*}\right)^{2}}\right]$ with $\left(E_{\mathrm{c}}^{*}\right)^{2}=\frac{3 m^{*} \hbar}{e^{2}\left(\tau_{2}^{*}\right)^{3}}$

and

$\left(\tau_{2}^{*}\right)^{3}=\frac{3}{4 \omega^{3}}\left(\sinh \left(2 \omega \tau_{2}\right)-2 \omega \tau_{2}\right)$.

The tunneling process is controlled by $\omega \tau_{2}$ where the tunneling time $\tau_{2}$ for $\varepsilon \ll \varepsilon_{\mathrm{T}}$ depends on the temperature like, Ref. [9],

$\tau_{2}=\hbar / 2 k T-\tau_{1}$.

Here $\tau_{1}$ is of the order of the period of the impurity vibration. At $\omega \tau_{2}$ much smaller than unity $\tau_{2}^{*} \simeq \tau_{2}$ and the ionization probability is independent on frequency. Lowering the temperature leads to an increase of $\tau_{2}$ according to Eq. (4) and in the same frequency range $\omega \tau_{2}$ becomes larger than unity. Now the ionization probability strongly depends on the laser frequency.

In AlGaAs:Te phonon-assisted tunneling has been observed over the entire range of available electric field strength. The ratio of the conductivity under irradiation to the dark conductivity $\sigma_{\mathrm{i}} / \sigma_{\mathrm{d}}$, which is proportional to the ionization probability $e(E) / e(0)$, is plotted in Fig. 2 as a function of the square of the peak electric field strength $E$. The ionization probability at $T=100 \mathrm{~K}$ (Fig. 2, top plate) is independent of the radiation frequency and increases with rising $E$ like $\exp \left(E^{2} / E_{\mathrm{c}}^{* 2}\right)$, according to Eqs. (2) and (3) with $\omega \tau_{2} \ll 1$. Such a behavior has also been

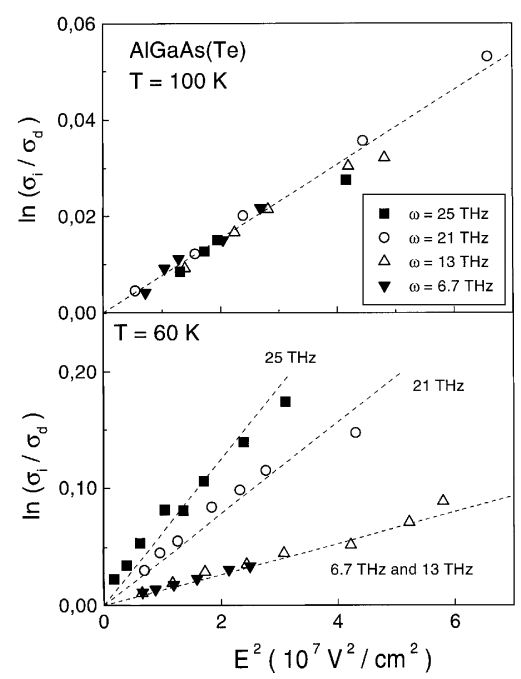

Fig. 2. Dependence of $\ln \left(\sigma_{\mathrm{i}} / \sigma_{\mathrm{d}}\right)$ on $E^{2}$ for $T=100$ and $T=60 \mathrm{~K}$. 


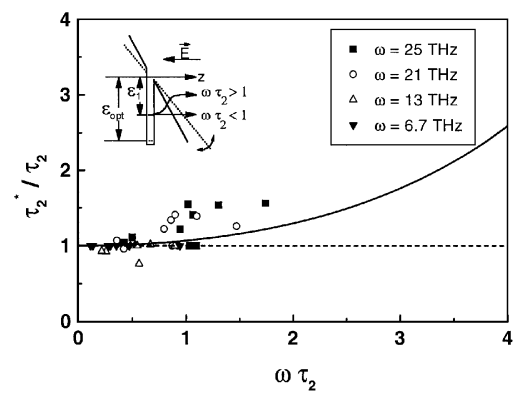

Fig. 3. Ratio $\tau_{2}^{*} / \tau_{2}$ as a function of $\omega \tau_{2}$. Experimentally obtained results are compared to calculations according to Eq. (2) (straight line). Inset: Electron tunneling in an electric field.

observed for other materials like DX-centers in AlGaSb : Te and substitutional impurities in germanium at sufficiently high temperatures, Refs. [5,8,9]. Lowering the temperature leads to an increase of $\tau_{2}$, given by Eq. (4), and thus in the same frequency range $\omega \tau_{2}$ becomes larger than unity. As shown in Fig. 2 (bottom plate) for $T=60 \mathrm{~K}$, the ionization probability gets frequency dependent and is drastically enhanced with rising frequency $\omega$. The ionization probability still depends exponentially on the square of the electric field strength, but the magnitude of $E_{c}^{*}$ decreases strongly with increasing frequency.

Varying the temperature and the radiation frequency the dependence of the effective time $\tau_{2}^{*}$ has been obtained from the measured values of $E_{\mathrm{c}}^{*}$ for various frequencies and temperatures. In Fig. 3, the ratio $\tau_{2}^{*} / \tau_{2}$ determined experimentally is plotted as a function of $\omega \tau_{2}$ and compared to calculations after Eq. (2).

In order to get even larger values of $\omega \tau_{2}$ measurements at the temperature of liquid helium have been carried out. Because of the large binding energy of the DX-center, the sample resistance is too high to detect any signal at this temperature. Therefore, persistent photoconductivity has been used to reduce the sample resistance by several orders of magnitude by illumination with visible and near-infrared light. After this illumination has been switch off, positive persistent photoconductivity could be detected with FIR-radiation. Fig. 4 shows experimental results at $T=4.2 \mathrm{~K}$ in the frequency range between 3.8 and $25 \mathrm{THz}$. In order to display in one figure the total set of data covering seven orders of magnitude in the square of the electric field, $E^{2}$ has been plotted on the abscissa in a logarithmic scale. To make an easy comparison with the $\exp \left(E^{2} / E_{\mathrm{c}}^{* 2}\right)$ dependence of $\sigma_{\mathrm{i}} / \sigma_{\mathrm{d}}$ possible, a logarithmic presentation of $\ln \left(\sigma_{\mathrm{i}} / \sigma_{\mathrm{d}}\right)$ has been used for the ordinate.

At $4.2 \mathrm{~K}$ and at low field strengths the condition $\omega \tau_{2} \gg 1$ is valid for the whole frequency range investigated here. The measurements of Fig. 4 show that at liquid helium temperature in the limit of $\omega \tau_{2} \gg 1$ a dras-

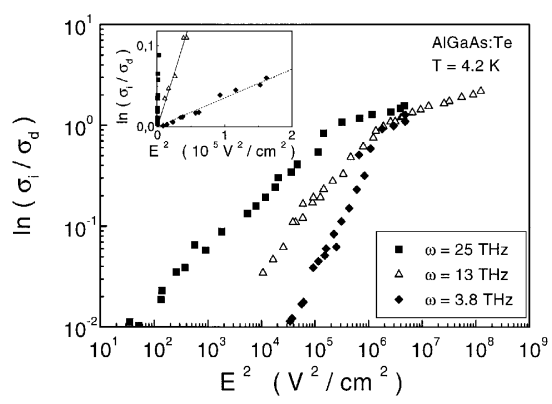

Fig. 4. Dependence of $\ln \left(\sigma_{\mathrm{i}} / \sigma_{\mathrm{d}}\right)$ on $E^{2}$ for $T=4.2 \mathrm{~K}$, the inset shows the low field behavior.

tic frequency dependence is observed. For a given constant signal a change of three orders of magnitude of electric fields squared needs only a six-times change in frequency. Note, that this large variation in the field strength yielding the same signal is valid only for the case of relatively low fields. As shown in the inset of Fig. 4, in this regime the ionization probability for the two lowest frequencies $\omega=3.8$ and $\omega=13 \mathrm{THz}$ can be described in terms of phonon-assisted tunneling $e(E) \propto \exp \left(E^{2} / E_{\mathrm{c}}^{* 2}\right)$ as it was the case for higher temperatures. At higher field strength the field dependence of the emission probability is much weaker and the frequency dependence practically disappears.

These changes of the dependence of the ionization probability on the field strength and radiation frequency is a result of the transition from phonon-assisted tunneling at low field strengths to direct tunneling without involving phonons at high fields, Ref. [5]. The emission probability for phonon-assisted tunneling as a function of the electric field strength given by Eqs. (2) and (3) was obtained in the limit that corrections to thermal emission resulting from electron tunneling are small, i.e. the electron tunneling energy is much smaller than the thermal ionization energy, $\varepsilon \ll \varepsilon_{\mathrm{T}}$. In the opposite limit, direct carrier tunneling from the ground state into continuum, without participation of phonons, becomes dominant. Direct electron tunneling occurs, if the potential $U_{2 \varepsilon}$ crosses $U_{1}$ in its minimum, where an electronic transition is possible without any change in the configuration coordinate. This effect, leading to weaker growth of the ionization probability in comparison with the field dependence of phonon-assisted tunneling, extrapolated to higher field strengths, determines the ionization process at very high fields, Refs. [1,5].

In Fig. 5 solid lines show the result of calculations for AlGaAs : Te at $4.2 \mathrm{~K}$ and frequencies used in the experiments taking into account both processes, phononassisted tunneling and direct tunneling, but ignoring the change of carrier density due to pre-illumination. 


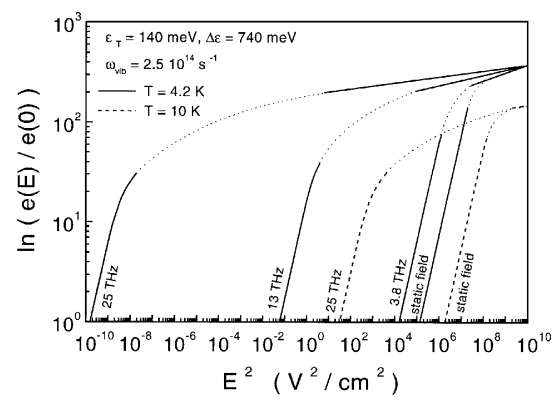

Fig. 5. Calculations of phonon-assisted and direct tunneling for $T=4.2$ and $T=10 \mathrm{~K}$.

Because of singularities, calculations at $\omega \tau_{2} \gg 1$ have been carried out in limited and separated ranges of the electric field strength and the gaps have been interpolated. The theory qualitatively describes well the experimentally observed features of the field and frequency dependence of tunneling ionization. The disappearance of the frequency effects at very high fields can be explained by the electric field dependence of the tunneling time $\tau_{2}$. In the high field regime the defect tunneling trajectory is shifted to lower energies, as shown in Fig. 1, leading to a decrease of $\tau_{2}$. By this $\omega \tau_{2}$ becomes smaller than unity and the frequency dependence vanishes.

Theoretical expressions for tunneling ionization have been obtained under the condition that after excitation electrons and phonons remain in thermal equilibrium at liquid helium temperature. In experiment, pre-illumination and persistent photoconductivity has been used to increase the free carrier density by several orders of magnitude. Thus free electrons may be heated by FIRradiation due to Drude absorption. To achieve quantitative agreement of theory and experiment, heating of the phonon system by energy transfer from the electrons should be taken into account. An increase of the sample temperature of just a few degrees leads to lower relative emission rates $e(E) / e(0)$ and a much weaker frequency dependence of the emission probability as demonstrated in Fig. 5 by broken lines.

\section{Acknowledgements}

Financial support by the DFG and the NATO Linkage Grant is gratefully acknowledged. S.D.G. and I.N.Y. wish to thank the RFFI grant 98-02-18268, H.K. the Hans-Böckler-Stiftung and E.Z. the Graduiertenkolleg Komplexität in Festkörpern.

\section{References}

[1] V.N. Abakumov, V.I. Perel, I.N. Yassievich, Nonradiative Recombination in Semiconductors, in: V.M. Agranovich, A.A. Maradudin (Eds.), Modern Problems in Condensed Matter Sciences, Vol. 33, North-Holland, Amsterdam, 1991.

[2] P.T. Landsberg, Recombination in Semiconductors, Cambridge University Press, Cambridge, 1991.

[3] H.J. von Bardeleben, I. Buyanova, A. Belyaev, M. Sheinkman, Phys. Rev. B 45 (1992) 11667.

[4] A.E. Belyaev, Yu.S. Ryabchenko, M.K. Sheinkman, H.J. von Bardeleben, Semicond. Sci. Technol. 11 (1996) 68.

[5] S.D. Ganichev, W. Prettl, I.N. Yassievich, Phys. Solid State 39 (1997) 1703.

[6] P.M. Mooney, T.N. Theis, Comments Cond. Mat. Phys. 16 (1992) 167.

[7] R.C. Newman, Semicond. Sci. Technol. 9 (1994) 1749.

[8] S.D. Ganichev, E. Ziemann, Th. Gleim, W. Prettl, I.N. Yassievich, V.I. Perel, I. Wilke, E.E. Haller, Phys. Rev. Lett. 80 (1998) 2409.

[9] S.D. Ganichev, J. Diener, I.N. Yassievich, W. Prettl, B.K. Meyer, K.W. Benz, Phys. Rev. Lett. 75 (1995) 1590. 